\title{
Outcomes of emergency and interval hepatectomy for ruptured resectable hepatocellular carcinoma: a single tertiary referral centre experience
}

\author{
Andrew Kai Yip Fung, Charing Ching Ning Chong, Kit Fai Lee, John Wong, Yue Sun Cheung, Anthony Kwong Wai \\ Fong, Paul Bo San Lai
}

Department of Surgery, the Chinese University of Hong Kong, Prince of Wales Hospital, Hong Kong 999077, Hong Kong, China.

Correspondence to: Dr. Andrew Kai Yip Fung, Department of Surgery, The Chinese University of Hong Kong, Prince of Wales Hospital, Shatin, Hong Kong 999077, Hong Kong, China. E-mail: andrewfung@surgery.cuhk.edu.hk

How to cite this article: Fung AKY, Chong CCN, Lee KF, Wong J, Cheung YS, Fong AKW, Lai PBS. Outcomes of emergency and interval hepatectomy for ruptured resectable hepatocellular carcinoma: a single tertiary referral centre experience. Hepatoma Res 2017;3:196-204.

Article history:

Received: 24-05-2017

Accepted: 29-08-2017

Published: 08-09-2017

Key words:

Hepatocellular carcinoma,

rupture,

hepatectomy

\begin{abstract}
Aim: The short and long term outcomes of patients who underwent emergency and interval hepatectomy for ruptured and resectable hepatocellular carcinoma (HCC) were analysed. Methods: The data of patients with ruptured HCC presenting between April 2004 and October 2015 were analysed. Emergency hepatectomy was defined as hepatectomy within $48 \mathrm{~h}$ of the clinico-radiological diagnosis of HCC rupture. Results: Thirty patients underwent hepatectomy for ruptured HCC. Nine (30\%) patients underwent emergency hepatectomy. The median age was 56 and 54 years $(P=0.13)$ with a similar gender distribution. The mean HCC size $(10.5$ vs. $8.3 \mathrm{~cm}, P=0.17)$, total blood loss $(3,000$ vs. $850 \mathrm{~mL}, P=0.002)$ and total units of red blood cell transfusion ( 1.9 vs. 0.5 units, $P=0.27$ ) were greater in the emergency hepatectomy group. The complication rate was $44 \%$ and $38 \%(P=0.53)$, with median length of hospital stay of 10 and 12 days $(P=0.07)$ in the emergency and interval hepatectomy groups, respectively, and no 30-day mortality in both groups. The median overall survival was 29 and 15.7 months $(P=0.25)$, with survival rates of $78 \%, 45 \%, 0 \%$ and $85 \%, 43 \%$ and $5 \%$ at 1,3 and 5 years in the emergency and interval hepatectomy groups, respectively. Conclusion: Hepatectomy should be considered for ruptured HCC provided the patient could tolerate curative resection.
\end{abstract}

\section{INTRODUCTION}

Hepatocellular carcinoma ( $\mathrm{HCC}$ ) is the fifth commonest malignancy globally. ${ }^{[1]}$ Rupture of $\mathrm{HCC}$ is the third commonest presentation of this condition, with an incidence of $3-15 \%$ and an associated in-hospital mortality of up to $75 \% \cdot{ }^{[2-4]}$ The pathogenesis of $\mathrm{HCC}$ rupture includes increased pressure within the tumour, rapid tumour growth or necrosis. This situation might be exacerbated by the presence of liver cirrhosis with concurrent thrombocytopenia and coagulopathy. ${ }^{[5]}$

The treatment for ruptured HCC is determined by the haemodynamic stability of the patient. ${ }^{[6]}$ In the

cc) () 9 (2) This is an open access article distributed under the terms of the Creative Commons AttributionCC) NonCommercial-ShareAlike 3.0 License, which allows others to remix, tweak, and build upon the work non-commercially, as long as the author is credited and the new creations are licensed under the identical terms.




presence of haemodynamic stability, non-operative management with close monitoring is gold standard care. ${ }^{[7]}$ However, when there is haemodynamic instability, several treatment options are available. These include non-operative procedures such as transarterial embolisation or absolute alcohol injection, and surgical intervention (perihepatic packing, hepatic artery ligation, suture ligation, radiofrequency ablation or hepatic resection). ${ }^{[8-12]}$ Despite the multiple treatment options for ruptured HCC, there remains no consensus on the optimal approach for these patients.

The advantage of one stage emergency liver resection is the spontaneous control of haemorrhage with definitive management of the HCC. Emergent operation can also reduce the duration of peritoneal seeding of ruptured HCC tumour cells by lavage with water at operation. ${ }^{[13,14]}$

However, emergent operative intervention must be balanced against the high mortality rate of up to $40 \%{ }^{[15]}$ consequent to the lack of pre-operative objective assessment of functional liver reserve and extent of disease burden, hypovolaemic shock condition and coagulopathy. ${ }^{[16]}$

As a result, the alternative option of staged liver resection after initial haemorrhage control with trans-arterial embolisation (TAE) is offered in some centres. This allows for subsequent assessment of functional liver reserve and operation under elective circumstances. The success of TAE haemostasis is $50-100 \%$, with a risk of liver failure of up to $33 \%$. Additionally, the 30 day mortality after TAE is lower compared to emergent hepatectomy (0-9\% vs. 0-37\%). ${ }^{[16]}$

The survival benefits of two-stage liver resection over emergent hepatectomy remain controversial. Liu et al..15] concluded that survival after two-stage liver resection post-HCC rupture was inferior compared to patients who did not have this complication, whereas Yeh et al. ${ }^{[17]}$ found that ruptured HCC had similar overall survival rates compared to non-ruptured HCC but inferior disease-free survival rates. Mizuno et al. ${ }^{[18]}$ noted that there was no difference in overall survival between ruptured and non-ruptured HCC.

In this retrospective single-centre study, the short and long term outcomes of patients who underwent emergency and interval hepatectomy for ruptured and resectable $\mathrm{HCC}$ were analysed.

\section{METHODS}

Patients with a diagnosis of ruptured HCC presenting between April 2004 and October 2015 to our hospital were retrieved from the in-house prospectively maintained hepatectomy database. The clinical data of these patients were collected and analysed retrospectively. In addition, the hepatectomy histopathology results were reviewed to confirm HCC rupture.

Due to the prospective nature of the database, some patients had just undergone hepatectomy and had not had sufficient follow-up period so were excluded for data analysis. Patients with intra-operative findings of incidental peri-tumoural haematoma suggestive of previously ruptured HCC were excluded. Emergency hepatectomy was defined as liver resection within $48 \mathrm{~h}$ of the clinical or radiological diagnosis of HCC rupture. Some patients were referred to our hospital after haemodynamic stabilisation at the parent hospital using TAE. These patients were included in the emergency hepatectomy group if they proceeded to liver resection within $48 \mathrm{~h}$ of first presentation of HCC rupture.

TAE was performed by experienced interventional radiologists with selective cannulation and then embolisation of the tumour-feeding artery with gelfoam particles. Surgical intervention was indicated when TAE failed to achieve adequate haemostasis. An experienced team of hepato-biliary surgeons performed hepatectomy. Hepatic parenchymal transection was undertaken using an ultrasonic dissector and TissueLink (Medtronic, Ireland) radiofrequency dissector. Intermittent Pringle manoeuvre might be applied during hepatectomy. The clinical decision algorithm for ruptured HCC as utilised in the author's institution is shown in Figure 1.

Post-operative follow-up of hepatectomy included ultrasound at 3 months and contrast triphasic computed tomography (CT) at 6 months with 3 -monthly monitoring of serum alpha-fetoprotein and liver function test for 2 years, then 6-monthly thereafter. Supplementary CT was done in the presence of raised serum alpha-fetoprotein or suspicion of $\mathrm{HCC}$ recurrence on ultrasound. Recurrent $\mathrm{HCC}$ was diagnosed with radiological imaging (CT or positron emission tomography $\mathrm{CT}$ ) to identify the location of intra-hepatic recurrence, tumour disease burden and the presence of extra-hepatic disease recurrence. Treatment options for recurrent HCC included further liver resection, local ablation therapies, transarterial chemo-embolisation (TACE), external beam radiotherapy, systemic chemotherapy or targeted immunotherapy. A multi-disciplinary team decided on treatment, taking into account the patients' liver functional status, recurrence pattern and comorbidities. 


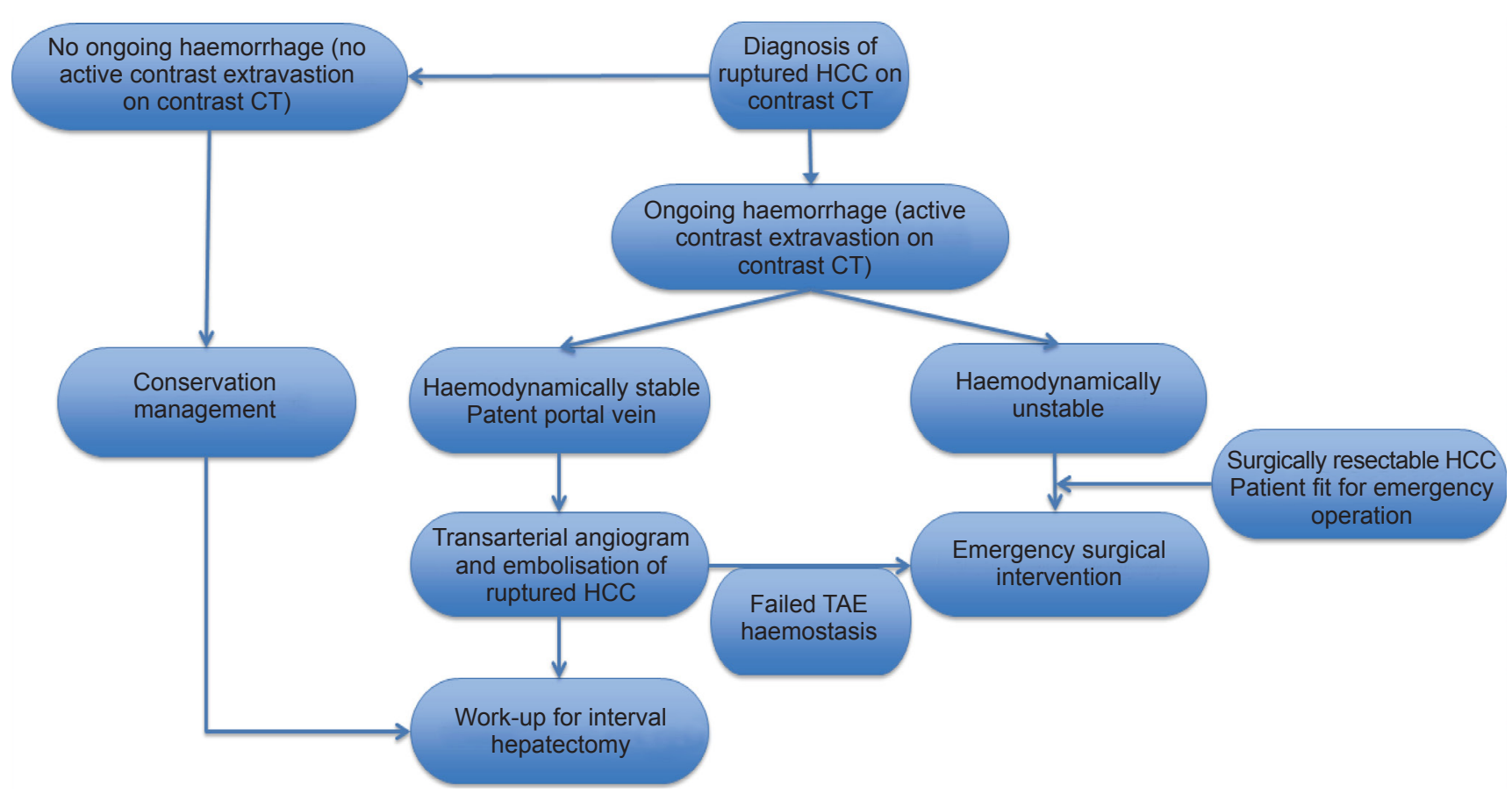

Figure 1: Algorithm for the management of ruptured HCC. HCC: hepatocellular carcinoma; TAE: trans-arterial embolisation; CT: computed tomography

Statistical analysis was performed with independent $t$ test for continuous variables and chi-square test was used to compare discrete variables. Kaplan-Meier analysis was used to estimate overall survival between emergency and interval hepatectomy groups. Overall survival was defined as the time from hepatectomy until death from any cause, or until the observation period was completed. Survival data were censored on November 7th, 2015. Statistical significance was defined as a $P$ value $<0.05$ and statistical calculations were performed on SPSS 22 software (IBM).

\section{RESULTS}

\section{Preoperative demographics}

Thirty patients underwent hepatectomy for ruptured resectable HCC. Nine (30\%) patients underwent emergency hepatectomy with a median time to operation of 0 days (range $0-2$ ). For interval hepatectomy, median time to operation was 19 days (range 3-49). The median age for patients who underwent emergency hepatectomy was 56 years compared to 54 years in the interval hepatectomy group $(P=0.13)$. There was a similar distribution of male patients in both groups $(89 \%$ vs. $90 \%, P=0.66)$. The pre-operative haemoglobin $(10.1$ vs. $12.0, P=$ $0.07)$ and platelet count (171 vs. $220, P=0.11)$ were lower and creatinine was worse (102 vs. 87, $P=0.32$ ) in the emergency hepatectomy group but this did not reach statistical significance. There were no significant differences in pre-operative international normalized ratio and bilirubin levels between the two groups.

Eight (89\%) and 18 (90\%) patients in the emergency and interval hepatectomy groups were hepatitis B virus positive, respectively $(P=0.66)$. There was more severe liver dysfunction in the emergency hepatectomy group, with higher pre-operative Child-Pugh grade $(P=$ 0.04 , Table 1).

Five $(56 \%)$ patients underwent pre-operative TAE in the emergency hepatectomy group compared to 10 patients $(48 \%)$ in the interval hepatectomy group $(P=$ $0.5)$. Two patients in the emergency group had failed embolisation due to small collateral vessels, whereas 4 patients had unresponsive shock despite adequate fluid resuscitation and proceeded to emergency hepatectomy without prior TAE.

\section{Operative characteristics}

In the emergency hepatectomy group, all patients underwent anatomical resection (5 left lateral sectionectomies, 2 left hepatectomies and 2 right hepatectomies) compared to $15(76 \%)$ in the interval group (3 left lateral sectionectomies, 2 left hepatectomies, 9 right hepatectomies and 1 caudate lobectomy) $(P=0.07)$. The mean $\mathrm{HCC}$ tumour size was larger $(10.5$ vs. $8.3 \mathrm{~cm}, P=0.17)$ in the emergency hepatectomy group.

The mean operative time for liver resection in the 
Table 1: Patient demographics and intraoperative characteristics, expressed as means with standard deviation or median with range

\begin{tabular}{|c|c|c|c|}
\hline & $\begin{array}{l}\text { Emergency hepatectomy } \\
\qquad(n=9)\end{array}$ & $\begin{array}{l}\text { Interval hepatectomy } \\
((n=21)\end{array}$ & $P$ value \\
\hline Male & $8(89 \%)$ & $18(90 \%)$ & 0.66 \\
\hline Age (years) & $56 \pm 15$ & $54 \pm 10$ & 0.13 \\
\hline ASA at time of operation & & & 0.19 \\
\hline 1 & - & 1 & \\
\hline 2 & 4 & 13 & \\
\hline 3 & 2 & 6 & \\
\hline 4 & 3 & 1 & \\
\hline Pre-operative haemoglobin ( $\mathrm{g} / \mathrm{L})$ & $10.1 \pm 3.5$ & $12.0 \pm 2.0$ & 0.07 \\
\hline Platelet count & $171 \pm 67$ & $220 \pm 71$ & 0.11 \\
\hline INR & $1.3 \pm 0.5$ & $1.1 \pm 0.2$ & 0.10 \\
\hline Bilirubin & $16 \pm 13$ & $15 \pm 11$ & 0.78 \\
\hline Creatinine & $102 \pm 43$ & $87 \pm 33$ & 0.32 \\
\hline Hepatitis B carrier & $8(89 \%)$ & $18(90 \%)$ & 0.66 \\
\hline Hepatitis C carrier & $1(11 \%)$ & $1(5 \%)$ & 0.93 \\
\hline AFP & $2,790(2-23,400)$ & $3,220(2-32,500)$ & 0.89 \\
\hline Child-Pugh A cirrhosis & 4 & 17 & 0.04 \\
\hline Child-Pugh B cirrhosis & 3 & 4 & \\
\hline Child-Pugh $\mathrm{C}$ cirrhosis & 2 & 0 & \\
\hline Prior trans-arterial angiogram and embolisation & $5(56 \%)$ & $10(48 \%)$ & 0.50 \\
\hline Failed embolisation of ruptured $\mathrm{HCC}$ & $2(22 \%)$ & - & \\
\hline Time from diagnosis of rupture $\mathrm{HCC}$ to liver resection (days) & $0(0-2)$ & $25(3-49)$ & $<0.05$ \\
\hline Anatomical resection & $9(100 \%)$ & $15(71 \%)$ & 0.07 \\
\hline Operative time (min) & $200 \pm 71$ & $276 \pm 83$ & 0.02 \\
\hline Blood loss: skin incision to start of hepatectomy $(\mathrm{mL})$ & $1,900 \pm 1,130$ & $390 \pm 250$ & 0.012 \\
\hline Start to finish of hepatectomy $(\mathrm{mL})$ & $630 \pm 490$ & $305 \pm 250$ & 0.06 \\
\hline Total operative blood loss ( $\mathrm{mL})$ & $3,000 \pm 1,500$ & $850 \pm 440$ & 0.002 \\
\hline Blood transfusion post-op (units) & $1.3 \pm 0.5$ & $1.3 \pm 0.5$ & 0.86 \\
\hline Total blood transfusion (units) & $1.9 \pm 3.6(0-11)$ & $0.5 \pm 0.8(0-2)$ & 0.27 \\
\hline Duration of drain placement (days) & $5(3-10)$ & $4(3-11)$ & 0.83 \\
\hline Cirrhosis & $6(67 \%)$ & $15(71 \%)$ & 0.10 \\
\hline Ishak liver cirrhosis scores $(0-6)$ & $4 \pm 2(1-6)$ & $5 \pm 2(0-6)$ & 0.31 \\
\hline Tumour size $(\mathrm{cm})$ & $10.5 \pm 4.3$ & $8.3 \pm 3.8$ & 0.17 \\
\hline Number of tumour lesions & & & 0.58 \\
\hline 1 & 6 & 14 & \\
\hline 2 & - & 3 & \\
\hline 3 & 1 & 1 & \\
\hline 4 & - & 1 & \\
\hline$>5$ & 2 & 2 & \\
\hline Micro-vascular invasion & $6(67 \%)$ & $11(52 \%)$ & 0.52 \\
\hline Resection margin $(\mathrm{cm})$ & $1.3 \pm 1.1(0.5-4.0)$ & $1.7 \pm 1.1(0-4.5)$ & 0.47 \\
\hline
\end{tabular}

ASA: American Society of Anesthesiologists; INR: international normalized ratio; AFP: alpha-fetoprotein; HCC: hepatocellular carcinoma

emergency and interval hepatectomy groups were $200 \pm 71$ and $276 \pm 83$ min respectively $(P=0.02)$. Total blood loss $(3,000$ vs. $850 \mathrm{~mL}, P=0.002)$ and the mean total units of red blood cell transfusion (1.9 vs. 0.5 units, $P=0.27$ ) were greater in the emergency hepatectomy group [Table 2].

\section{Post-operative outcomes}

The post-operative complication rate was $44 \%$ and $38 \%$ in the emergency and interval hepatectomy groups respectively $(P=0.53)$. One patient in the interval hepatectomy group required pigtail drainage of pleural effusion. The median total length of hospital stay was 10 and 12 days respectively $(P=0.07)$ with no 30-day mortality in both groups [Table 2].
The median time to intra-hepatic recurrence was 7.8 months in the emergency hepatectomy group and 5.0 months in the interval hepatectomy group $(P=0.12)$. The median time to extra-hepatic recurrence was 6.8 and 9.7 months $(P=0.59)$, to earliest recurrence was 6.8 and 5.6 months $(P=0.74$, Figure $2 A)$ and overall survival was 29 and 15.7 months $(P=0.25$, Figure 2B) respectively. Survival rates were $78 \%, 45 \%, 0 \%$ and $85 \%, 43 \%$ and $5 \%$ at 1,3 and 5 years in the emergency and interval hepatectomy groups respectively [Table 2].

In the present study, patients who underwent emergency hepatectomy had more pulmonary recurrence (33\% vs. $19 \%$ ) compared to the interval group at followup. Additionally, the time to intra-hepatic recurrence 
Table 2: Post-operative outcomes and long-term follow-up data for post-hepatectomy patients, expressed as medians with range

\begin{tabular}{|c|c|c|c|}
\hline & Emergency hepatectomy $(n=9)$ & Interval hepatectomy $(n=21)$ & $P$ value \\
\hline \multicolumn{4}{|l|}{ Complications } \\
\hline Wound infection & 2 & 2 & 0.35 \\
\hline Pleural effusion & 2 & 3 & 0.60 \\
\hline Pleural effusion requiring drainage & 0 & 1 & 0.51 \\
\hline Confusion & 0 & 1 & 0.12 \\
\hline Ascites & 0 & 1 & 0.51 \\
\hline Total & $4(44 \%)$ & $8(38 \%)$ & 0.53 \\
\hline Median hospital stay after hepatectomy (days) & $10(5-17)$ & $12(6-32)$ & 0.07 \\
\hline 30 day mortality rate & 0 & 0 & - \\
\hline Time to intra-hepatic recurrence (months) & $7.8(2.6-100)$ & $5.0(1.1-39.5)$ & 0.12 \\
\hline Time to extra-hepatic recurrence (months) & $6.8(6.4-8.9)$ & $9.7(4.0-47.9)$ & 0.59 \\
\hline Peritoneal recurrence & $1(11 \%)$ & $6(29 \%)$ & 0.27 \\
\hline Time to peritoneal recurrence (months) & 6.4 & $6.4(4.0-10.1)$ & 0.55 \\
\hline Pulmonary recurrence & $3(33 \%)$ & $4(19 \%)$ & 0.44 \\
\hline Time to pulmonary recurrence (months) & $6.8(6.4-8.9)$ & $7.9(4.0-12.2)$ & 0.06 \\
\hline Recurrence in other location & 0 & $4(33 \%)$ & 0.15 \\
\hline Time to other location recurrence (months) & - & $11.7(10.1-47.8)$ & \\
\hline Time to earliest recurrence (months) & 6.8 & 5.6 & 0.74 \\
\hline Overall survival (months) & $29(4-100)$ & $15.7(8-49)$ & 0.25 \\
\hline 1-year overall survival & $7 / 9(78 \%)$ & $18 / 21(85 \%)$ & 0.59 \\
\hline 3-year overall survival & $4 / 9(45 \%)$ & $9 / 21(43 \%)$ & 0.94 \\
\hline 5-year overall survival & $0 / 9(0 \%)$ & $1 / 21(5 \%)$ & 0.51 \\
\hline
\end{tabular}


Figure 2: (A) Recurrence-free survival after emergency and interval hepatectomy for ruptured hepatocellular carcinoma $(P=0.74$, log rank test); (B) overall survival after emergency and interval hepatectomy for ruptured hepatocellular carcinoma $(P=0.25$, log rank test)

was longer, but extra-hepatic recurrence shorter in the emergency hepatectomy group. Overall median survival time was longer in the emergency group (29 vs. 15.7 months, $P=0.26$ ) but overall 1 -, 3 - and 5-year survival rates were similar in both groups.

\section{DISCUSSION}

Rupture of hepatocellular carcinoma $(\mathrm{HCC})$ is a rare but life-threatening complication of $\mathrm{HCC}$, and is associated with a high mortality rate (up to $75 \%$ ) in the acute phase due to a combination of hypovolemic shock, coagulopathy and subsequent hepatic failure. ${ }^{[19,20]}$
The risk factors for HCC rupture are multifactorial, and include rapid tumour growth with necrosis, vessel erosion or venous thrombosis by tumour cells. ${ }^{[16,21]}$ Additionally, left lobe tumours might be more inclined to rupture due to the smaller anatomical span of the left lobe. ${ }^{[22]}$

Bassi et al. ${ }^{[23]}$ commented that rupture of HCC which were located at the free surfaces of the liver can result in bleeding into the peritoneal cavity due to the lack of hepatic parenchyma covering the tumour. Kanematsu et al. ${ }^{[24]}$ showed that tumour protrusion was a risk factor for its subsequent rupture, whereas 
Li et al. ${ }^{[25]}$ identified tumours located in segments II, III and $\mathrm{VI}$ to be associated with its rupture. Furthermore, tumour rupture can occur in both large and small HCCs. ${ }^{[8]}$ Chan et al. ${ }^{[26]}$ found that ruptured HCC was associated with more aggressive disease compared to non-ruptured HCC as evidenced by higher tumour marker titres, higher rates of micro-vascular invasion and tumour multifocality. Zhu et al. ${ }^{[5]}$ found that tumour size $>5 \mathrm{~cm}$, hypertension, liver cirrhosis, vascular thrombus and extra-hepatic invasion were predictive of spontaneous HCC rupture on multivariate analysis.

In the present study, emergency hepatectomy was defined as liver resection within $48 \mathrm{~h}$ of the clinical or radiological diagnosis of $\mathrm{HCC}$ rupture. In the published literature, there are no guidelines on the optimal time for emergency operative intervention for ruptured HCC. Whilst an arbitrary method to distinguish hepatectomy into same admission liver resection (emergency group), and hepatectomy during second hospitalization (elective) is valid and clinically practical, we undertook this subgroup analysis and found that the there was considerable overlap between emergency and interval hepatectomy groups in terms of the time interval from onset of ruptured HCC to liver resection (data not shown). However, the use of the 48-h time interval resulted in eliminated this overlap.

The indications for emergency hepatectomy comprised of patients with CT confirmed ruptured HCC that presented with hypovolaemic shock, which was refractory to adequate fluid resuscitation and with failed trans-arterial angiogram and embolization of the ruptured HCC. Patients who remained haemodynamically unstable for angiogram were transferred to the operating room. The liver function and CT were assessed for feasibility of safe and curative hepatectomy prior to proceeding with emergency operation.

The patients who underwent emergency hepatectomy had worse preoperative Child-Pugh grade, larger tumour size, greater operative blood loss and blood transfusion requirements and higher rates of anatomical resection but shorter operative times compared to the elective hepatectomy group. In the post-operative period, the complication rate was higher in the emergency group (44\% vs. $38 \%$ ) but there were no 30 -day mortality or requirement for re-operative intervention in both groups. Emergency hepatectomy for ruptured HCC in patients with Child-Pugh C cirrhosis is associated with significant peri-operative mortality as reported in other case series, ${ }^{[20,23,27]}$ but in this present study, 2 patients with Child-Pugh $\mathrm{C}$ cirrhosis underwent emergency hepatectomy without 30-day mortality. The favourable post-operative outcomes might be related to the short operation time (mean $146 \mathrm{~min}$ ), small transection area (mean $35 \mathrm{~cm}^{2}$ ) and no pre-operative angiogram and embolisation.

The main objective of ruptured HCC treatment is haemorrhage control whilst preserving as much functional liver tissue as possible. ${ }^{[6,28,29]}$ The management of ruptured $\mathrm{HCC}$ is challenging and multiple treatment options are available, dependent on the clinical condition and haemodynamic stability of the patient. ${ }^{[7]}$

TAE is the preferred method for non-operative haemostasis of ruptured HCC. ${ }^{[20,30]}$ TAE can function as definitive palliative therapy or act as a bridge to interval hepatectomy. ${ }^{[23,27,31]}$ However, whilst TAE may achieve haemostasis of the tumour haemorrhage, there are risks of re-bleeding, liver abscess and this intervention cannot treat the tumour cells that have seeded the peritoneal cavity. ${ }^{[32]}$ Surgical intervention for ruptured HCC is indicated when haemostasis with TAE has been unsuccessful. ${ }^{[16]}$

Yang et al. ${ }^{[33]}$ reviewed the outcomes of 132 patients with ruptured HCC, of which 17 patients underwent emergency hepatectomy and 11 patients had TAE then interval hepatectomy. There were no 30-day mortality and 1-year survival rates were $56.3 \%$ and $63.6 \%$ respectively. The median overall survival was 13.0 and 14.6 months. In the present series, 1-year survival was $78 \%$ and $85 \%$, with overall median survival of 29 months in the emergency hepatectomy group compared to 15.7 months in the interval group $(P=0.25)$.

Zhang et al. ${ }^{[29]}$ reported on the impact of interval hepatectomy or repeat TACE after successful TACE for ruptured HCC. One hundred and twenty-six cases of ruptured $\mathrm{HCC}$ underwent TAE for haemostasis of which 74 had interval hepatectomy. The 90 -day mortality rate was $6.8 \%$ in the hepatectomy group and $7.7 \%$ in the TACE group $(P=0.84)$, all of whom died from tumour recurrence. The 1-, 3-, 5-year survival rates were $85.1 \%, 63.5 \%$ and $37.8 \%$ in the hepatectomy group compared to $69.2 \%, 46.2 \%$ and $17.3 \%$ in the TACE group $(P=0.004)$.

Dissemination of ruptured HCC tumour cells into the peritoneal cavity is one argument for proponents of emergency hepatectomy for ruptured HCC. ${ }^{[34,35]}$ Zhang et al. ${ }^{[29]}$ reported an $11.8 \%$ incidence of peritoneal disease in their series of ruptured HCCs. In the present study, there was an $11 \%$ peritoneal recurrence rate in the emergency hepatectomy group compared to $29 \%$ 
in the interval hepatectomy group $(P=0.27)$. The mean time to peritoneal recurrence was 6.4 and 6.4 months $(P=0.55)$ in the emergency and interval hepatectomy groups respectively. This $11 \%$ peritoneal recurrence rate was similar to that of hepatectomy for non-ruptured $\mathrm{HCC}$ as reported by Jianyong et al. ${ }^{[36]}$ In Chan et al. ${ }^{[26]}$ of interval hepatectomy for ruptured HCC, they found an intra-hepatic recurrence rate of $23.8 \%$ and extrahepatic recurrence rate of $17.9 \%(n=77)$. Additionally, peritoneal recurrence was $14.9 \%$ compared to $9.9 \%$ in a matched non-ruptured HCC group $(P=0.5)$. Hiraoka et al. ${ }^{[37]}$ found a peritoneal recurrence rate of $7.7 \%$ in their case series. Other researchers have also noted no increase in the incidence of peritoneal metastases after ruptured HCC. ${ }^{[18,38]}$ Moreover, there are reports to suggest that patients with peritoneal recurrence after hepatectomy for HCC have no prior evidence of HCC rupture. ${ }^{[39]}$ These results suggested that intra-peritoneal tumour cell implantation might not be a common event. Although peritoneal recurrence of $\mathrm{HCC}$ can be managed by radical surgical resection, in the present case series, all the patients with resectable peritoneal recurrence opted for non-surgical treatments.

In this study, the median time to extrahepatic recurrence was shorter in the emergency hepatectomy group, with no statistical difference in overall survival. There were no statistical differences in the tumour size, vascular involvement, resection margins or degree of cirrhosis, to explain the mechanisms for earlier extrahepatic recurrence in the emergency hepatectomy group (data not shown). Whether there is increased haematogenous spread of HCC tumour cells at the time of emergency compared to interval hepatectomy with subsequent extrahepatic seeding and HCC recurrence is a concept that this study cannot answer.

Yang et al. ${ }^{[40]}$ reported on the outcomes of 143 patients who underwent emergency $(n=28)$ or interval hepatectomy $(n=115)$ for ruptured HCC. Interestingly, they found that the recurrence-free survival $(23 \%, 9 \%$ and $9 \%$ vs. $45 \%, 26 \%$ and $16 \%$ at 1,3 and 5 years, $P=$ $0.025)$ and overall survival ( $50 \%, 8 \%$ and $8 \%$ vs. $70.3 \%$, $29.2 \%$ and $19.4 \%$ at 1,3 and 5 years, $P=0.016$ ) were worse in the emergency group. This data suggested that the ruptured HCC tumours were advanced at the time of presentation with probable micro-metastases. Although the median overall survival time was longer in the emergency group, the absolute numbers in this group were small which might skew the data and give a false survival advantage in the emergency group.

There were several limitations in this study. This was a retrospective analysis of patients with ruptured and resectable $\mathrm{HCC}$ managed at a single tertiary referral centre. The absolute number of patients was low given the rarity of rupture HCCs, although all eligible patients for analysis were included. There was selection bias in determining which patients should proceed to interval hepatectomy for ruptured $\mathrm{HCC}$ with the prerequisite of satisfactory liver functional reserve and resectable $\mathrm{HCC}$ s with curative intent. The heterogeneous nature of patient and tumour characteristics was another potential source of bias. Furthermore, the departmental database focussed on patients who underwent hepatectomy, and consequently, the data and clinical outcomes for patients who had ruptured HCC but were not subjected to hepatectomy (i.e. managed with TAE only or best supportive care) cannot be retrieved for analysis.

In conclusion, this study showed the feasibility of emergency or interval hepatectomy for highly selected patients with ruptured and resectable HCC. Although patients in the emergency hepatectomy group had larger tumours, worse pre-operative Child's grading and greater intra-operative blood loss, the recurrence-free and overall survival rates were similar in both groups. Hepatectomy should be considered for ruptured HCC provided the patient could tolerate curative resection and have surgically resectable tumours.

\section{DECLARATIONS}

\section{Authors' contributions}

Data collection, compiling results, writing and producing the final manuscript: A.K.Y. Fung

Editing the manuscript drafts: C.C.N. Chong, K.F. Lee, J. Wong, Y.S. Cheung, A.K.W. Fong, P.B.S. Lai

Approved the final manuscript for submission: A.K.Y. Fung, C.C.N. Chong, K.F. Lee, J. Wong, Y.S. Cheung, A.K.W. Fong, P.B.S. Lai

\section{Financial support and sponsorship}

None.

\section{Conflicts of interest}

There are no conflicts of interest.

\section{Patient consent}

The data obtained through the medical record review were managed according to the privacy policy and ethics code of our institute.

\section{Ethics approval}

This was a retrospective study and did not require Institutional Review Board approval. 


\section{REFERENCES}

1. Jemal A, Bray F, Center MM, Ferlay J, Ward E, Forman D. Global cancer statistics. CA Cancer J Clin 2011;61:69-90.

2. Tarantino L, Sordelli I, Calise F, Ripa C, Perrotta M, Sperlongano P. Prognosis of patients with spontaneous rupture of hepatocellular carcinoma in cirrhosis. Updates Surg 2011;63:25-30.

3. Kim JY, Lee JS, Oh DH, Yim YH, Lee HK. Transcatheter arterial chemoembolization confers survival benefit in patients with a spontaneously ruptured hepatocellular carcinoma. Eur J Gastroenterol Hepatol 2012;24:640-5.

4. Hsueh KC, Fan HL, Chen TW, Chan DC, Yu JC, Tsou SS, Chang TM, Hsieh CB. Management of spontaneously ruptured hepatocellular carcinoma and hemoperitoneum manifested as acute abdomen in the emergency room. World J Surg 2012;36:2670-6.

5. Zhu Q, Li J, Yan JJ, Huang L, Wu MC, Yan YQ. Predictors and clinical outcomes for spontaneous rupture of hepatocellular carcinoma. World $J$ Gastroenterol 2012;18:7302-7.

6. Yoshida H, Mamada Y, Taniai N, Uchida E. Spontaneous ruptured hepatocellular carcinoma. Hepatol Res 2016;46:13-21.

7. Ribeiro Junior MA, Chaib E, Saad WA, D'Albuquerque LA, Cecconello I. Surgical management of spontaneous ruptured hepatocellular adenoma. Clinics (Sao Paulo) 2009;64:775-9.

8. Battula N, Madanur M, Priest O, Srinivasan P, O'Grady J, Heneghan MA, Bowles M, Muiesan P, Heaton N, Rela M. Spontaneous rupture of hepatocellular carcinoma: a Western experience. Am J Surg 2009;197:164-7.

9. Chen ZY, Qi QH, Dong ZL. Etiology and management of hemmorrhage in spontaneous liver rupture: a report of 70 cases. World J Gastroenterol 2002;8:1063-6.

10. Yoshida H, Mamada Y, Taniai N, Mizuguchi Y, Kakinuma D, Ishikawa Y, Kanda T, Matsumoto S, Bando K, Akimaru K, Tajiri T. Long-term results of elective hepatectomy for the treatment of ruptured hepatocellular carcinoma. J Hepatobiliary Pancreat Surg 2008; 15:178-82.

11. Leowardi C, Hormann Y, Hinz U, Wente MN, Hallscheidt P, Flechtenmacher C, Buchler MW, Friess H, Schwarzbach MH. Ruptured angiosarcoma of the liver treated by emergency catheterdirected embolization. World J Gastroenterol 2006;12:804-8.

12. Cheung TT, Poon RT, Chok KS, Chan AC, Tsang SH, Dai WC, Yau TC, Chan SC, Fan ST, Lo CM. Management of spontaneously ruptured hepatocellular carcinomas in the radiofrequency ablation era. PLoS One 2014;9:e94453.

13. Sonoda T, Kanematsu T, Takenaka K, Sugimachi K. Ruptured hepatocellular carcinoma evokes risk of implanted metastases. J Surg Oncol 1989;41:183-6.

14. Yeh CN, Chen HM, Chen MF, Chao TC. Peritoneal implanted hepatocellular carcinoma with rupture after TACE presented as acute appendicitis. Hepatogastroenterology 2002;49:938-40.

15. Liu CL, Fan ST, Lo CM, Tso WK, Poon RT, Lam CM, Wong J. Management of spontaneous rupture of hepatocellular carcinoma: single-center experience. J Clin Oncol 2001;19:3725-32.

16. Lai EC, Lau WY. Spontaneous rupture of hepatocellular carcinoma: a systematic review. Arch Surg 2006;141:191-8.

17. Yeh CN, Lee WC, Jeng LB, Chen MF, Yu MC. Spontaneous tumour rupture and prognosis in patients with hepatocellular carcinoma. $\mathrm{Br} \mathrm{J}$ Surg 2002;89:1125-9.

18. Mizuno S, Yamagiwa K, Ogawa T, Tabata M, Yokoi H, Isaji S, Uemoto S. Are the results of surgical treatment of hepatocellular carcinoma poor if the tumor has spontaneously ruptured? Scand J Gastroenterol 2004;39:567-70.

19. Anon C. Delayed versus emergency hepatectomy for ruptured hepatocellular carcinoma. Indian J Surg 2006;68:209-15.
20. Tanaka A, Takeda R, Mukaihara S, Hayakawa K, Shibata T, Itoh K, Nishida N, Nakao K, Fukuda Y, Chiba T, Yamaoka Y. Treatment of ruptured hepatocellular carcinoma. Int J Clin Oncol 2001;6:291-5.

21. Chearanai O, Plengvanit U, Asavanich C, Damrongsak D, Sindhvananda K, Boonyapisit S. Spontaneous rupture of primary hepatoma: report of 63 cases with particular reference to the pathogenesis and rationale treatment by hepatic artery ligation. Cancer 1983;51:1532-6.

22. Chen CY, Lin XZ, Shin JS, Lin CY, Leow TC, Chen CY, Chang TT. Spontaneous rupture of hepatocellular carcinoma. A review of 141 Taiwanese cases and comparison with nonrupture cases. J Clin Gastroenterol 1995;21:238-42.

23. Bassi N, Caratozzolo E, Bonariol L, Ruffolo C, Bridda A, Padoan L, Antoniutti M, Massani M. Management of ruptured hepatocellular carcinoma: implications for therapy. World $J$ Gastroenterol 2010;16:1221-5.

24. Kanematsu M, Imaeda T, Yamawaki Y, Seki M, Goto H, Sone Y, Inuma G, Mochizuki R, Doi H. Rupture of hepatocellular carcinoma: predictive value of CT findings. AJR Am J Roentgenol 1992;158:124750 .

25. Li J, Huang L, Liu CF, Cao J, Yan JJ, Xu F, Wu MC, Yan YQ. Risk factors and surgical outcomes for spontaneous rupture of BCLC stages A and B hepatocellular carcinoma: a case-control study. World J Gastroenterol 2014;20:9121-7.

26. Chan AC, Dai JW, Chok KS, Cheung TT, Lo CM. Prognostic influence of spontaneous tumor rupture on hepatocellular carcinoma after interval hepatectomy. Surgery 2016;159:409-17.

27. Lin HM, Lei LM, Zhu J, Li GL, Min J. Risk factor analysis of perioperative mortality after ruptured bleeding in hepatocellular carcinoma. World J Gastroenterol 2014;20:14921-6.

28. Casciaro GE, Spaziani E, Costantino A, Ceci F, Di Grazia C, Martellucci A, Pecchia M, Cipriani B, De Angelis F, Corelli S, Napoleoni A, Stefanelli F, Salvadori C, Parisella M, Nicodemi $\mathrm{S}$, Stagnitti F. Liver resection for hemoperitoneum caused by spontaneous rupture of unrecognized hepatocellular carcinoma. $G$ Chir 2012;33:221-4.

29. Zhang DZ, Zhang K, Wang XP, Cai H. Patients with spontaneously ruptured hepatocellular carcinoma benefit from staged surgical resection after successful transarterial embolization. Asian Pac $J$ Cancer Prev 2015;16:315-9.

30. Shimada R, Imamura H, Makuuchi M, Soeda J, Kobayashi A, Noike T, Miyagawa S, Kawasaki S. Staged hepatectomy after emergency transcatheter arterial embolization for ruptured hepatocellular carcinoma. Surgery 1998;124:526-35.

31. Chu F, Morris DL. Single centre experience of liver resection for hepatocellular carcinoma in patients outside transplant criteria. Eur $J$ Surg Oncol 2006;32:568-72.

32. Hai L, Yong-Hong P, Yong F, Ren-Feng L. One-stage liver resection for spontaneous rupture of hepatocellular carcinoma. World J Surg 2005;29:1316-8.

33. Yang H, Chen K, Wei Y, Liu F, Li H, Zhou Z, Li B. Treatment of spontaneous ruptured hepatocellular carcinoma: a single-center study. Pak J Med Sci 2014;30:472-6.

34. Shuto T, Hirohashi K, Kubo S, Tanaka H, Hamba H, Kubota D, Kinoshita H. Delayed hepatic resection for ruptured hepatocellular carcinoma. Surgery 1998;124:33-7.

35. Lin CH, Hsieh HF, Yu JC, Chen TW, Yu CY, Hsieh CB. Peritoneal lavage with distilled water during liver resection in patients with spontaneously ruptured hepatocellular carcinomas. J Surg Oncol 2006;94:255-6.

36. Jianyong L, Jinjing Z, Wentao W, Lunan Y, Qiao Z, Bo L, Tianfu W, Minqing X, Jiaying Y, Yongang W. Preoperative transcatheter arterial chemoembolization for resectable hepatocellular carcinoma: a single 
center analysis. Ann Hepatol 2014;13:394-402.

37. Hiraoka A, Kawamura T, Aibiki T, Okudaira T, Toshimori A, Yamago H, Nakahara H, Suga Y, Azemoto N, Miyata H, Miyamoto Y, Ninomiya T, Murakami T, Ishimaru Y, Kawasaki H, Hirooka M, Abe M, Matsuura B, Hiasa Y, Michitaka K. Prognosis and therapy for ruptured hepatocellular carcinoma: problems with staging and treatment strategy. Eur J Radiol 2015;84:366-71.

38. Lee HS, Choi GH, Kang DR, Han KH, Ahn SH, Kim DY, Park JY, Kim SU, Choi JS. Impact of spontaneous hepatocellular carcinoma rupture on recurrence pattern and long-term surgical outcomes after partial hepatectomy. World J Surg 2014;38:2070-8.

39. Lin CC, Liang HP, Lee HS, Huang GT, Yang PM, Ho MC, Lee PH, Tsang YM, Chen DS, Sheu JC, Chen CH. Clinical manifestations and survival of hepatocellular carcinoma patients with peritoneal metastasis. J Gastroenterol Hepatol 2009;24:815-20.

40. Yang T, Sun YF, Zhang J, Lau WY, Lai EC, Lu JH, Shen F, Wu MC. Partial hepatectomy for ruptured hepatocellular carcinoma. Br J Surg 2013;100:1071-9. 